Review

\title{
Mechanisms of Membrane Curvature Generation in Membrane Traffic
}

\section{Hye-Won Shin ${ }^{1,2, *}$, Hiroyuki Takatsu ${ }^{1}$ and Kazuhisa Nakayama ${ }^{2}$}

1 Career-Path Promotion Unit for Young Life Scientists, Kyoto University, Sakyo-ku, Kyoto 606-8501, Japan; E-Mail: takachu@cp.kyoto-u.ac.jp

2 Graduate School of Pharmaceutical Sciences, Kyoto University, Sakyo-ku, Kyoto 606-8501, Japan; E-Mail: kazunaka@pharm.kyoto-u.ac.jp

* Author to whom correspondence should be addressed; E-Mail: shin@cp.kyoto-u.ac.jp; Tel.: +81-757-539-306; Fax: +81-757-539-311.

Received: 29 January 2012; in revised form: 20 February 2012 / Accepted: 21 February 2012 / Published: 29 February 2012

\begin{abstract}
During the vesicular trafficking process, cellular membranes undergo dynamic morphological changes, in particular at the vesicle generation and fusion steps. Changes in membrane shape are regulated by small GTPases, coat proteins and other accessory proteins, such as BAR domain-containing proteins. In addition, membrane deformation entails changes in the lipid composition as well as asymmetric distribution of lipids over the two leaflets of the membrane bilayer. Given that P4-ATPases, which catalyze unidirectional flipping of lipid molecules from the exoplasmic to the cytoplasmic leaflets of the bilayer, are crucial for the trafficking of proteins in the secretory and endocytic pathways, changes in the lipid composition are involved in the vesicular trafficking process. Membrane remodeling is under complex regulation that involves the composition and distribution of lipids as well as assembly of proteins.
\end{abstract}

Keywords: membrane trafficking; small GTPase; BAR domain; P4-ATPase; membrane curvature 


\section{Introduction}

Biological membranes not only separate the interior of the cell from the external environment, but also bring about compartmentalization of distinct subcellular organelles. Intracellular compartments along the secretory and endocytic pathways communicate with one another through membrane trafficking, specifically that mediated by carrier vesicles. Membrane trafficking ensures delivery of appropriate materials, such as proteins and lipids, in order to maintain the identity of these organelles. Dynamic membrane shape changes occur during formation of the vesicles and tubules that mediate cargo delivery in the secretory and endocytic pathways. These carrier intermediates vary in shape, from small vesicles ( 50 nm) and thin tubules to tubular-saccular carriers [1-3].

Upon membrane deformation, a selected membrane subdomain is curved, sensed and stabilized by a variety of proteins. Formation of carrier intermediates is driven through a complex process that involves modification and changes in composition and distribution of lipids, as well as local assembly of coat protein complexes and proteins involved in sensing and forming membrane curvature. In particular, insertion of amphipathic helices into the membrane subdomain is required for curvature generation; proteins containing BAR (Bin/Amphiphysin/Rvs) domains, which form a crescent-like homodimer, are required for sensing and stabilizing this curvature. Here, we will introduce the idea of local curvature generation via changes in the inter-leaflet distribution of lipids, catalyzed by P4-ATPases, and insertion of the amphipathic helices of Arf family small GTPases. Furthermore, we will discuss how the curvature is stabilized by BAR domain proteins. We also consider the interplays among these proteins that generate and stabilize membrane curvature in the context of membrane trafficking.

\section{Lipids}

\subsection{Relationship between Asymmetric Lipid Distribution and Membrane Deformation}

Lipid bilayers, including those of the plasma membrane and the membrane-enclosed compartments along the secretory and endocytic pathways, exhibit asymmetric lipid distributions. In particular, aminophospholipids, phosphatidylserine (PS) and phosphatidylethanolamine (PE) are enriched in the cytoplasmic leaflet. For example, in resting human red blood cells, PS and PE are restricted primarily to the inner/cytoplasmic leaflet of the plasma membrane, whereas phosphatidylcholine (PC) and sphingomyelin (SM) are exposed on the cell surface [4,5]. Regulated exposure of PS in the outer/exoplasmic leaflet occurs during a variety of biological processes, such as apoptotic cell death, platelet coagulation and myotube formation [5-8]. On the other hand, PE is exposed on the surface of the cleavage furrow during cytokinesis [9].

Drastic cell shape changes occur upon exogenous application of lyso-PC or impermeable amphipathic drugs. The former flips into the inner leaflet of the bilayer at a slow rate; the latter intercalate only into the exterior leaflet of the bilayer; these phenomena can be explained by the bilayer couple mechanism [10,11]. Moreover, insertion of lyso-PC into a giant unilamellar vesicle induces formation of a single bud [12]. These observations suggest that changes in distribution of specific 
lipids between the two leaflets of bilayers are able to drive membrane deformation.

\subsection{Phospholipid Flippases (P4-ATPases)}

An ATP-dependent "flippase" (also called aminophospholipid translocase) activity in the plasma membrane of human erythrocytes was discovered by Seigneuret and Devaux [13]. Subsequently, P4-ATPases, a subfamily of P-type ATPases, were recognized as the most likely candidates for flippases in eukaryotic membranes [14-16]. P4-ATPases have been implicated in flipping aminophospholipids from the exoplasmic/lumenal leaflet to the cytoplasmic leaflet of cellular membranes [14,17-20]. In yeast, it has also been reported that even PC can be translocated by P4-ATPases [18].

Molecular biological approaches in yeasts and worm have revealed that the absence of specific P4-ATPases results in defects in membrane trafficking [14,15,18,21-24]. In the yeast Saccharomyces cerevisiae, five P4-ATPases are encoded in the genome (Table 1), and are involved in trafficking of proteins at different stages along the secretory and endocytic pathways [25]. For example, a drs2-ts mutant at the non-permissive temperature inhibits translocation of fluorescently labeled PS [26] and formation of post-Golgi vesicles [27], indicating that Drs2p is required to flip PS to the cytoplasmic leaflet of membranes in order to support vesicle formation from the trans-Golgi network (TGN). Alder-Baerens et al. [28] showed that loss of Drs2p and Dnf3p disrupts aminophospholipid translocation and phospholipid asymmetry in yeast post-Golgi secretory vesicles. In other organisms, including Caenorhabditis elegans and Arabidopsis thaliana, P4-ATPases also play essential roles in membrane trafficking [22,23,29]. For example, Poulsen et al. [29] showed that mutations of ALA3 (a P4-ATPase in A. thaliana) result in impaired production of secretory vesicles from the Golgi. These findings confirm the critical roles of lipid flippases in vesicle formation. P4-ATPases may induce membrane bending via changing the distribution of phospholipids between the two leaflets of bilayers (Figure 1A).

Table 1. P4-ATpases in human, yeast, worm and plant.

\begin{tabular}{|c|c|c|c|c|}
\hline Class & Human & S. cerevisiae & C. elegans & A. thaliana \\
\hline $1 \mathrm{a}$ & ATP8A1, ATP8A2 & DRS2 & TAT-1 & ALA1 \\
\hline $1 \mathrm{~b}$ & $\begin{array}{l}\text { АТР8В1, АТР8В2, } \\
\text { АТР8В3, АТР8В4 }\end{array}$ & - & TAT-2 & \multirow{6}{*}{ ALA2-12 } \\
\hline 2 & АТР9А, АТР9В & NEO1 & TAT-5, TAT-6 & \\
\hline 3 & - & DNF1, DNF2 & - & \\
\hline 4 & - & DNF3 & - & \\
\hline 5 & ATP10A, ATP10B, ATP10D & - & TAT-3, TAT-4 & \\
\hline 6 & ATP11A, ATP11B, ATP11C & - & - & \\
\hline
\end{tabular}


Figure 1. Mechanistic model for the generation of membrane curvature. (A) Membrane deforming mechanism (i) Lipid composition is changed by P4-ATPases, LPAT and PLA2; (ii) amphipathic helices of Arf family small GTPases are inserted into the cytoplasmic leaflet of the membrane; and (iii) the resultant curvature is sensed and stabilized by BAR domain proteins; (B) Helical-wheel representation of the N-terminal amphipathic helix of Arf1 (amino acids 2-13); (C) Structure of the BAR domain dimer of arfaptin. Image from the RCSB PDB [30] of PDB ID 1I49 [31]; (D) The complex regulation of proteins involved in generating membrane curvature.

A

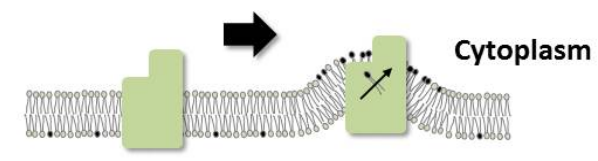

(i)
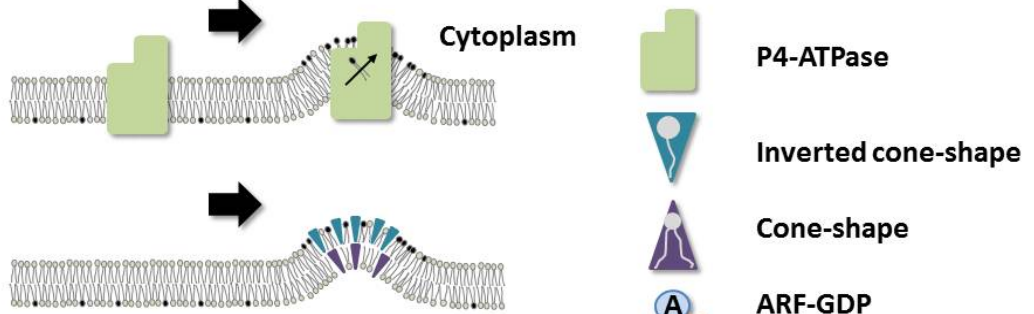

A. ARF-GDP

(A)

(ii)

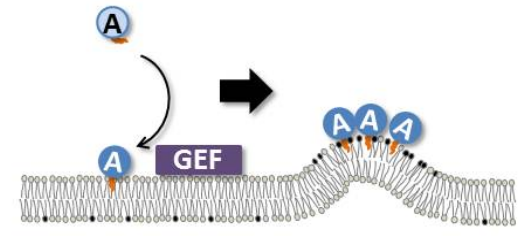

ARF-GTP

in $\mathrm{N}$-terminal helix

- BAR domain

(iii)

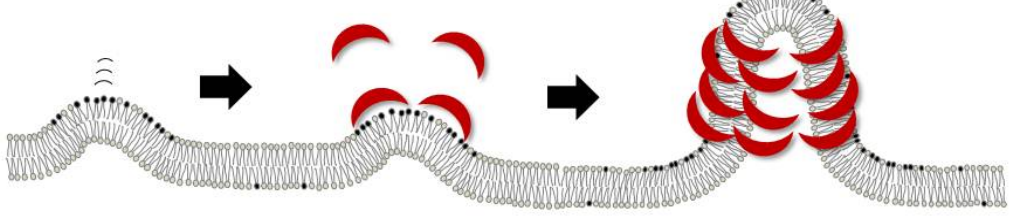

B

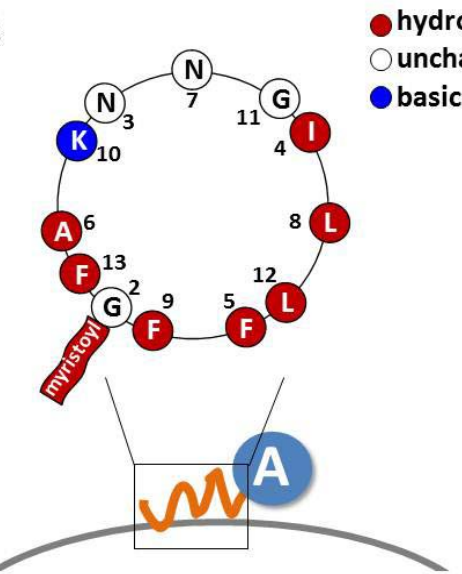

\section{C}

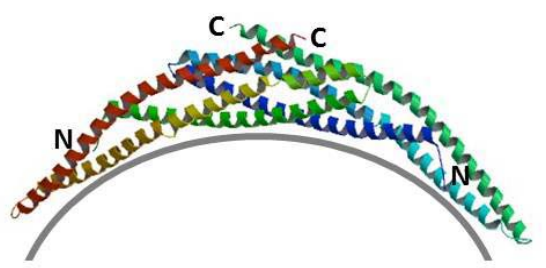

D

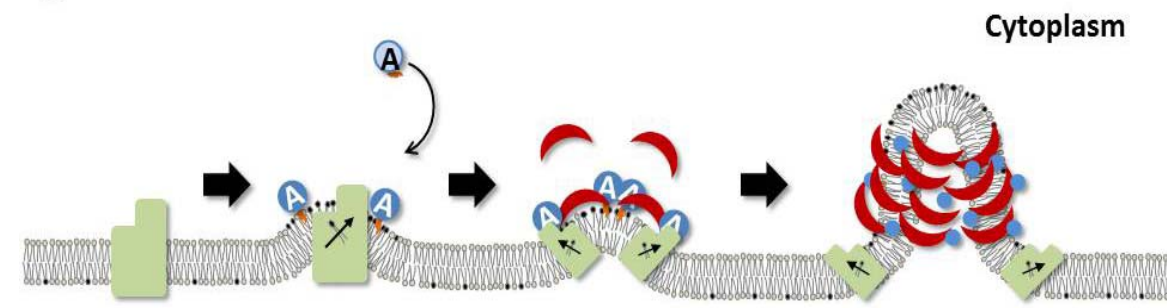


Although there has been no direct evidence indicating that mammalian P4-ATPases also play essential roles in membrane trafficking, earlier studies showed that changes in lipid composition are involved in vesicular trafficking in a human erythroleukemia cell line, K562 [32]: endocytosis is stimulated by extracellular application of PS or PE, but partially inhibited by the addition of lyso-PS, which cannot translocate across the plasma membrane [32]. Thus, it is likely that the plasma membrane phospholipid asymmetry produces a driving force for budding of endocytic vesicles in mammalian cells as well.

Mammalian P4-ATPases have been implicated in various pathophysiologic processes. Among the 14 P4-ATPases (Table 1), mutations of ATP8B1 (FIC1) have been reported to cause familial intrahepatic cholestasis [33]. Furthermore, in ATP8B1-deficient mice, a significant amount of PS was observed in bile after infusion of bile salts, suggesting that ATP8B1 deficiency causes exposure of PS in the outer leaflet of the apical plasma membrane of bile canalicular cells [34]. The ATP8B3 gene is expressed in spermatocytes and spermatids [35]. In ATP8B3-null sperm cells, PS exposure was detectable before capacitation; the aberrantly early exposure of PS may affect the capacitation process [35]. The mouse ATP10A gene (also called ATP10C) is linked to diet-induced obesity and type II diabetes phenotypes [36], and an ATP10D mutation shows linkage to a fat-prone phenotype in certain strains of mice [37,38]. Recently, ATP11C has been shown to participate in the B lymphocyte differentiation [39,40]. Together, these observations imply that the asymmetric distribution of phospholipids in biological membranes maintained/regulated by P4-ATPases plays crucial roles in mammalian pathophysiology. However, the involvement of mammalian P4-ATPases in membrane trafficking, and the functional relationship between the P4-ATPase-related pathophysiology and membrane trafficking, remain poorly understood.

\subsection{Lipid-Modifying Enzymes (LPAT and $\left.P L A_{2}\right)$}

The size and chemical properties of different acyl chains and head groups of phospholipids can affect membrane curvature (Figure 1A) [41,42]. Phosphatidic acid (PA) and lyso-PA are interconverted by phospholipase $\mathrm{A}_{2}\left(\mathrm{PLA}_{2}\right)$ and lyso-PA acyl transferase (LPAT) activities, respectively. These lipids favor opposite membrane curvatures; lyso-PA and PA can induce budding towards the cytoplasm when located in the cytoplasmic and exoplasmic leaflets, respectively [43-45] (Figure 1A). Inhibitors of $\mathrm{PLA}_{2}$ prevent formation of membrane tubules from the Golgi apparatus and endosomes, and consequently inhibit protein trafficking through these organelles [44]. Conversely, application of specific LPAT inhibitors enhances tubulation of the Golgi [46]. Thus, local changes in the shape of phospholipids can influence membrane deformation and thereby the formation of carrier intermediates.

\section{Proteins}

\subsection{Arf Family Small GTPases}

Small GTPases act as molecular switches that regulate activity and localization of proteins and thereby determine the spatial and temporal organization of many cellular processes, including 
membrane trafficking. In their GTP-bound active state, members of the Arf family of small GTPases promote budding of coated carrier vesicles, such as those coated by the COPI complex and the clathrin/AP-1 complex [47]. Members of the Arf family (Arf1-Arf6) are related to a broad set of small GTPases [48]. These relatives include Sar1 and a subfamily of Arf-like (Arl) proteins. Arf1, the best-characterized member of the Arf subfamily, facilitates recruitment of COPI onto Golgi membranes and clathrin to late Golgi and endosomal compartments, both through its direct interaction with heterotetrameric adaptor complexes (AP-1, AP-3, AP-4) and monomeric adaptors (GGA1-GGA3) [49-51] and through activating lipid-modifying enzymes, including phospholipase D and phosphatidylinositol-specific kinases [52-56]. These data indicate that Arf1 acts as a master regulator of coated vesicle formation.

Arf family proteins contain an N-terminal amphipathic helix and a myristoyl group that is usually attached to the $\mathrm{N}$ terminus (Figure 1A,B). Upon binding to GTP, the Arf molecule undergoes an extensive conformational change, during which the interswitch region displaces the N-terminal amphipathic helix from a hydrophobic pocket $[57,58]$. The exposed helix is in turn inserted into an adjacent lipid bilayer, and can induce membrane curvature (Figure 1A,B). Indeed, GTP-bound Arf and Sar1 molecules effectively tubulate liposomes, whereas GDP-bound forms do not [59-64]. In case of Sar1, the exposure of the amphipathic N-terminal helix triggers membrane curvature generation during budding of COPII-coated vesicles [60]. Mutations within the N-terminal amphipathic helix of Arf1 decrease the number of Arf1-induced tubules in vivo and its ability to tubulate liposomes in vitro [61,63]. Additional support of this idea is provided by the observation that many membrane-deforming proteins, such as endophilins [65,66], epsins [67] and amphiphysins [68,69], contain amphipathic helices that can be inserted into the lipid bilayers. These data support a mechanistic model in which insertion of N-terminal amphipathic helix of GTP-bound Arf proteins leads to bilayer bending upon vesicle budding (Figure 1A).

\subsection{BAR Domain-Containing Proteins}

The BAR domain protein superfamily members have been implicated in membrane traffic, actin cytoskeleton remodeling and signal transduction [69-71]. BAR domains constitute a helical homodimer [69]. Given that BAR domain homodimers intrinsically adopt curved structures (Figure 1C), and that some BAR domain proteins, such as arfaptin and amphiphysin, can tubulate liposomes in vitro $[68,69]$, it is likely that BAR domain proteins can generally sense and/or induce membrane curvature upon their recruitment from the cytosol onto the membrane surface [72-74]. Crystal structures, lipid-binding studies and liposome tubulation assays have validated the BAR domain-mediated membrane curvature model and expanded the superfamily; according to sequence and structural similarities, the family now comprises classical BAR domain proteins as well as N-BAR, F-BAR, I-BAR, BAR-PH, and PX-BAR proteins (see [73,75] for details). N-BAR domain proteins, such as amphiphysins and endophilins, have an N-terminal amphipathic helix, and may trigger membrane deformation via insertion into lipid bilayers. The BAR domain in turn senses curvature, and may thereby play roles in biogenesis of tubular organelles or the generation of endocytic vesicles or 
other carrier intermediates [65,69,76]. The observation of crystal structures and cryo-electron microscopic analyses suggest that BAR domains self-assemble into larger complexes, and that individual domains may be in contact in an organized manner and form BAR domain lattices [77-80]. F-BAR domain proteins, such as FCHo and FBP17, sense membrane curvature of clathrin-coated pits, oligomerize around the curved membranes, and ultimately induce invagination and tubulation of the plasma membrane during endocytosis $[73,77,80,81]$. BAR domains that sense, and presumably can generate curvature can be recruited or activated at buds, tubules or tubular-saccular structures during budding processes [74,82,83] (Figure 1A).

In general, BAR domains bind to acidic membrane surfaces via basic residues on their concave face; the binding mode is relatively nonspecific, as compared with other membrane-binding modules that recognize head groups of specific phospholipids [69]. Many BAR domains are found in conjunction with other membrane-binding modules, such as $\mathrm{PH}$ (pleckstrin homology) and PX (phox homology) domains, which define membrane-binding specificity, and thereby functional specificity, of the BAR domain proteins [70]. For instance, PH domains, which recognize specific phosphoinositides, are required for correct membrane association of BAR domain proteins, APPL1 and ASAP1 (an ArfGAP) [84,85]. Moreover, a subset of the sorting nexin (SNX) family proteins, including SNX1 and SNX9, contain phosphoinositide-binding PX domains in addition to their BAR domains. The PX domain of SNX9 binds to phosphatidylinositol 4,5-bisphosphate (PtdIns $\left.(4,5) \mathrm{P}_{2}\right)$, and thereby assists in targeting of this SNX-BAR to PtdIns $(4,5) \mathrm{P}_{2}$-enriched regions of endocytic pits [86-88]. In contrast, the PX domain of SNX1 associates with the early and late endosomal phosphoinositides, respectively phosphatidylinositol 3-phosphate and phosphatidylinositol 3,5-bisphosphate, helping to target this protein to maturing early endosomes [89-91]. Therefore, the PX-BAR-containing SNXs can be selectively targeted to high-curvature membrane domains of cellular compartments that are enriched in specific phosphoinositides, and in turn drive membrane tubulation in the context of tubule-based sorting [92-94].

\section{Interplay between Regulatory Proteins}

\subsection{Membrane Targeting of BAR Domain Proteins by Small GTPases}

The BAR domain protein arfaptin was originally identified as a binding partner of the Arf family of small GTPases. We have recently shown that Arl1 (Arf-like 1) interacts with the BAR domain of arfaptin in a GTP-dependent manner [95]. Arl1 is localized primarily to the trans side of the Golgi complex, where it plays a role in membrane trafficking [96-99]. Overexpression of arfaptin induces Golgi-derived membrane tubules, whereas exogenous expression of Arl1 itself neither generates membrane tubules nor enhances arfaptin-mediated tubulation. However, association of arfaptin with Golgi membranes is abolished by depletion of endogenous Arl1 by RNAi, and rescued by exogenous Arl1 expression, indicating that Arl1 is required for targeting arfaptin to Golgi membranes. Although it is not clear whether association of Arl1 (or other proteins) or lipid modification provides the initial cue for membrane deformation, it is clear that arfaptin molecules recruited onto Golgi membranes through 
interaction with Arl1 sense and stabilize membrane curvature, and thereby facilitate tubule formation, probably through oligomerization like other BAR domain proteins [77-80] (Figure 1D).

The early endosomal protein APPL1 interacts with Rab5 via its BAR and PH domains. This interaction is critical for targeting of APPL1 to endosomal membranes and APPL-mediated regulation of cell proliferation $[85,100]$.

Thus, in addition to lipid-binding modules, such as PH and PX domains, the Arf and Rab families of small GTPases also determine the temporally and spatially confined localization of BAR domain proteins.

\subsection{Arf Family Small GTPases and P4-ATPases}

The first indication that P4-ATPases are involved in membrane trafficking came from an Arf1 synthetic lethal screen in yeast, designed to discover proteins that function together with Arf in vesicle budding from the Golgi complex [15]. DRS2 was identified in this screen. Drs2p localizes predominantly to the TGN; Drs2p-deficient cells are morphologically very similar to clathrin mutants [15]. As described above, Arf GTPases play an essential role in recruiting vesicle coat proteins, such as the COPI complex and the clathrin/AP-1 complex, from the cytosol, in order to induce their assembly on Golgi membranes. Genetic interactions were found between $d r s 2$, arf and clathrin mutant alleles, but not between $d r s 2$ and COPI or COPII mutations. Consistent with these genetic interactions, clathrin-dependent pathways are strongly perturbed by loss of Drs2p, whereas COPI- and COPII-dependent protein trafficking pathways are unaffected [101,102]. Moreover, Drs2p has been shown to directly interact with Gea2p, a guanine nucleotide exchange factor for Arf GTPases [103]. Because the localizations of Gea2p, AP-1 and clathrin are not affected in drs2 cells [102,103], it seems that Drs2p is not required for the recruitment of these proteins, but rather might be involved in the membrane deformation process by flipping phospholipids at sites of vesicle budding.

On the other hand, Neo1p, which is phylogenetically close to mammalian ATP9A and ATP9B (Table 1), exhibits genetic and physical interactions with Ysl2p, a potential guanine nucleotide exchange factor for Arl1p [104,105]. We and others have previously shown that Arl1 is localized to the TGN, where it regulates the retrograde trafficking from endosomes to the Golgi complex in mammalian cells $[96,98,99]$. Moreover, we have recently found that arfaptin (a BAR domain protein; see above) is a downstream effector of Arl1 [95], suggesting involvement of Arl1 in the membrane deformation machinery during vesicular trafficking (Figure 1D). Given that Neo1p may play a role in membrane trafficking within the endosomal/Golgi system [105], and that human ATP9A and ATP9B localize to the TGN and endosomes [106], ATP9A and ATP9B might be functionally implicated in the post-Golgi membrane trafficking (Figure 1B).

\section{Conclusions}

The curvature generation process is divided into two stages. The first stage is membrane deformation. The deformation can be induced by any of the following mechanisms: (i) changes in lipid composition (shapes, species and numbers of phospholipids) between two leaflets of the bilayer; 
(ii) insertion of small hydrophobic or amphipathic protein domains into one of the two leaflets; and (iii) binding of specific protein domains with an intrinsically curved shape (e.g., BAR domains) to the membrane surface, forcing the membrane to assume the shape of the protein domain. The second stage is curvature sensing, i.e., the preferential recognition and stabilization of preexisting lipid topologies by protein domains with suitable curvature. We favor a model in which BAR domains function to sense and stabilize preexisting membrane curvature, rather than to induce curvature. However, the two functions are not mutually exclusive, and may occur simultaneously or in a complex fashion. Thus, generation of membrane curvature in the context of membrane trafficking and organelle dynamics is under complex regulation, involving changes in the composition and distribution of lipids, assembly of proteins, and the orchestrated activities of these regulatory proteins.

\section{Acknowledgements}

This work was supported in part by grants from the Ministry of Education, Culture, Sports, Science and Technology of Japan, the Special Coordination Fund for Promoting Science and Technology, the Targeted Proteins research program, the Takeda Science Foundation, and the Inamori Foundation.

\section{References}

1. Polishchuk, R.S.; Polishchuk, E.V.; Marra, P.; Alberti, S.; Buccione, R.; Luini, A.; Mironov, A.A. Correlative light-electron microscopy reveals the tubular-saccular ultrastructure of carriers operating between Golgi apparatus and plasma membrane. J. Cell Biol. 2000, 148, 45-58.

2. McMahon, H.T.; Gallop, J.L. Membrane curvature and mechanisms of dynamic cell membrane remodelling. Nature 2005, 438, 590-596.

3. Zimmerberg, J.; Kozlov, M.M. How proteins produce cellular membrane curvature. Nat. Rev. Mol. Cell Biol. 2006, 7, 9-19.

4. Schrier, S.L.; Zachowski, A.; Devaux, P.F. Mechanisms of amphipath-induced stomatocytosis in human erythrocytes. Blood 1992, 79, 782-786.

5. Zwaal, R.F.; Schroit, A.J. Pathophysiologic implications of membrane phospholipid asymmetry in blood cells. Blood 1997, 89, 1121-1132.

6. Van den Eijnde, S.M.; van den Hoff, M.J.; Reutelingsperger, C.P.; van Heerde, W.L.; Henfling, M.E.; Vermeij-Keers, C.; Schutte, B.; Borgers, M.; Ramaekers, F.C. Transient expression of phosphatidylserine at cell-cell contact areas is required for myotube formation. J. Cell Sci. 2001, 114, 3631-3642.

7. Williamson, P.; Schlegel, R.A. Transbilayer phospholipid movement and the clearance of apoptotic cells. Biochim. Biophys. Acta 2002, 1585, 53-63.

8. Leventis, P.A.; Grinstein, S. The distribution and function of phosphatidylserine in cellular membranes. Annu. Rev. Biophys. 2010, 39, 407-427.

9. Emoto, K.; Kobayashi, T.; Yamaji, A.; Aizawa, H.; Yahara, I.; Inoue, K.; Umeda, M. Redistribution of phosphatidylethanolamine at the cleavage furrow of dividing cells during cytokinesis. Proc. Natl. Acad. Sci. USA 1996, 93, 12867-12872. 
10. Sheetz, M.P.; Singer, S.J. Biological membranes as bilayer couples. A molecular mechanism of drug-erythrocyte interactions. Proc. Natl. Acad. Sci. USA 1974, 71, 4457-4461.

11. Devaux, P.F.; Herrmann, A.; Ohlwein, N.; Kozlov, M.M. How lipid flippases can modulate membrane structure. BBA-Biomembranes 2008, 1778, 1591-1600.

12. Papadopulos, A.; Vehring, S.; Lopez-Montero, I.; Kutschenko, L.; Stockl, M.; Devaux, P.F.; Kozlov, M.; Pomorski, T.; Herrmann, A. Flippase activity detected with unlabeled lipids by shape changes of giant unilamellar vesicles. J. Biol. Chem. 2007, 282, 15559-15568.

13. Seigneuret, M.; Devaux, P.F. ATP-dependent asymmetric distribution of spin-labeled phospholipids in the erythrocyte membrane: Relation to shape changes. Proc. Natl. Acad. Sci. USA 1984, 81, 3751-3755.

14. Tang, X.; Halleck, M.S.; Schlegel, R.A.; Williamson, P. A subfamily of P-type ATPases with aminophospholipid transporting activity. Science 1996, 272, 1495-1497.

15. Chen, C.Y.; Ingram, M.F.; Rosal, P.H.; Graham, T.R. Role for Drs2p, a P-type ATPase and potential aminophospholipid translocase, in yeast late Golgi function. J. Cell Biol. 1999, 147, 1223-1236.

16. Gomes, E.; Jakobsen, M.K.; Axelsen, K.B.; Geisler, M.; Palmgren, M.G. Chilling tolerance in arabidopsis involves ALA1, a member of a new family of putative aminophospholipid translocases. Plant Cell 2000, 12, 2441-2454.

17. Daleke, D.L. Regulation of transbilayer plasma membrane phospholipid asymmetry. J. Lipid Res. 2003, 44, 233-242.

18. Pomorski, T.; Lombardi, R.; Riezman, H.; Devaux, P.F.; van Meer, G.; Holthuis, J.C.M. Drs2p-related P-type ATPases Dnf1p and Dnf2p are required for phospholipid translocation across the yeast plasma membrane and serve a role in endocytosis. Mol. Biol. Cell 2003, 14, $1240-1254$.

19. Saito, K.; Fujimura-Kamada, K.; Furuta, N.; Kato, U.; Umeda, M.; Tanaka, K. Cdc50p, a protein required for polarized growth, associates with the Drs2p P-Type ATPase implicated in phospholipid translocation in saccharomyces cerevisiae. Mol. Biol. Cell 2004, 15, 3418-3432.

20. Zhou, X.; Graham, T.R. Reconstitution of phospholipid translocase activity with purified Drs2p, a type-IV P-type ATPase from budding yeast. Proc. Natl. Acad. Sci. USA 2009, 106, 16586-16591.

21. Zachowski, A.; Henry, J.P.; Devaux, P.F. Control of transmembrane lipid asymmetry in chromaffin granules by an ATP-dependent protein. Nature 1989, 340, 75-76.

22. Anne-Francoise, R.; Lars, N.; Fabrice, R.; Morten Krog, L.; Jean-Louis, B.; Simon, T. The C. elegans P4-ATPase TAT-1 regulates lysosome biogenesis and endocytosis. Traffic 2009, 10, 88-100.

23. Chen, B.; Jiang, Y.; Zeng, S.; Yan, J.; Li, X.; Zhang, Y.; Zou, W.; Wang, X. Endocytic sorting and recycling require membrane phosphatidylserine asymmetry maintained by TAT-1/CHAT-1. PLoS Genet. 2010, 6, e1001235. 
24. Wehman, A.M.; Poggioli, C.; Schweinsberg, P.; Grant, B.D.; Nance, J. The P4-ATPase TAT-5 inhibits the budding of extracellular vesicles in C. elegans embryos. Curr. Biol. 2011, 21, 1951-1959.

25. Graham, T.R. Flippases and vesicle-mediated protein transport. Trends Cell Biol. 2004, 14, 670-677.

26. Natarajan, P.; Wang, J.; Hua, Z.; Graham, T.R. Drs2p-coupled aminophospholipid translocase activity in yeast Golgi membranes and relationship to in vivo function. Proc. Natl. Acad. Sci. USA 2004, 101, 10614-10619.

27. Gall, W.E.; Geething, N.C.; Hua, Z.; Ingram, M.F.; Liu, K.; Chen, S.I.; Graham, T.R. Drs2p-dependent formation of exocytic clathrin-coated vesicles in vivo. Curr. Biol. 2002, 12, 1623-1627.

28. Alder-Baerens, N.; Lisman, Q.; Luong, L.; Pomorski, T.; Holthuis, J.C.M. Loss of P4 ATPases Drs2p and Dnf3p disrupts aminophospholipid transport and asymmetry in yeast post-Golgi secretory vesicles. Mol. Biol. Cell 2006, 17, 1632-1642.

29. Poulsen, L.R.; Lopez-Marques, R.L.; McDowell, S.C.; Okkeri, J.; Licht, D.; Schulz, A.; Pomorski, T.; Harper, J.F.; Palmgren, M.G. The arabidopsis P4-ATPase ALA3 localizes to the Golgi and requires a \{beta\}-Subunit to function in lipid translocation and secretory vesicle formation. Plant Cell 2008, 20, 658-676.

30. Protein Data Bank, Research Collaboratory for Structural Bioinformatics (RCSB PDB) web site. Available online: www.pdb.org (accessed on 29 February 2012).

31. Tarricone, C.; Xiao, B.; Justin, N.; Walker, P.A.; Rittinger, K.; Gamblin, S.J.; Smerdon, S.J. The structural basis of Arfaptin-mediated cross-talk between Rac and Arf signalling pathways. Nature 2001, 411, 215-219.

32. Farge, E.; Ojcius, D.M.; Subtil, A.; Dautry-Varsat, A. Enhancement of endocytosis due to aminophospholipid transport across the plasma membrane of living cells. Am. J. Physiol. Cell Physiol. 1999, 276, C725-733.

33. Paulusma, C.C.; Oude Elferink, R.P.J. The type 4 subfamily of P-type ATPases, putative aminophospholipid translocases with a role in human disease. BBA-Mol. Basis Dis. 2005, 1741, $11-24$.

34. Paulusma, C.C.; Groen, A.; Kunne, C.; Ho-Mok, K.S.; Spijkerboer, A.L.; Rudi de Waart, D.; Hoek, F.J.; Vreeling, H.; Hoeben, K.A.; van Marle, J.; et al. Atp8b1 deficiency in mice reduces resistance of the canalicular membrane to hydrophobic bile salts and impairs bile salt transport. Hepatology 2006, 44, 195-204.

35. Wang, L.; Beserra, C.; Garbers, D.L. A novel aminophospholipid transporter exclusively expressed in spermatozoa is required for membrane lipid asymmetry and normal fertilization. Dev. Biol. 2004, 267, 203-215.

36. Dhar, M.S.; Sommardahl, C.S.; Kirkland, T.; Nelson, S.; Donnell, R.; Johnson, D.K.; Castellani, L.W. Mice heterozygous for Atp10c, a putative amphipath, represent a novel model of obesity and type 2 diabetes. J. Nutr. 2004, 134, 799-805. 
37. Folmer, D.E.; Elferink, R.P.J.O.; Paulusma, C.C. P4 ATPases-Lipid flippases and their role in disease. BBA-Mol. Cell Biol. L. 2009, 1791, 628-635.

38. Flamant, S.; Pescher, P.; Lemercier, B.; Clément-Ziza, M.; Képès, F.; Fellous, M.; Milon, G.; Marchal, G.; Besmond, C. Characterization of a putative type IV aminophospholipid transporter P-type ATPase. Mamm. Genome 2003, 14, 21-30.

39. Siggs, O.M.; Arnold, C.N.; Huber, C.; Pirie, E.; Xia, Y.; Lin, P.; Nemazee, D.; Beutler, B. The P4-type ATPase ATP11C is essential for B lymphopoiesis in adult bone marrow. Nat. Immunol. 2011, 12, 434-440.

40. Yabas, M.; Teh, C.E.; Frankenreiter, S.; Lal, D.; Roots, C.M.; Whittle, B.; Andrews, D.T.; Zhang, Y.; Teoh, N.C.; Sprent, J.; et al. ATP11C is critical for the internalization of phosphatidylserine and differentiation of B lymphocytes. Nat. Immunol. 2011, 12, 441-449.

41. Hammond, K.; Reboiras, M.D.; Lyle, I.G.; Jones, M.N. Characterisation of phosphatidylcholine/ phosphatidylinositol sonicated vesicles. Effects of phospholipid composition on vesicle size. Biochim. Biophys. Acta 1984, 774, 19-25.

42. Sprong, H.; van der Sluijs, P.; van Meer, G. How proteins move lipids and lipids move proteins. Nat. Rev. Mol .Cell Biol. 2001, 2, 504-513.

43. Scales, S.J.; Scheller, R.H. Cell biology: Lipid membranes shape up. Nature 1999, 401, 123-124.

44. Brown, W.J.; Chambers, K.; Doody, A. Phospholipase A2 (PLA2) enzymes in membrane trafficking: Mediators of membrane shape and function. Traffic 2003, 4, 214-221.

45. Schmidt, J.A.; Brown, W.J. Lysophosphatidic acid acyltransferase 3 regulates Golgi complex structure and function. J. Cell Biol. 2009, 186, 211-218.

46. Drecktrah, D.; Chambers, K.; Racoosin, E.L.; Cluett, E.B.; Gucwa, A.; Jackson, B.; Brown, W.J. Inhibition of a Golgi complex lysophospholipid acyltransferase induces membrane tubule formation and retrograde trafficking. Mol. Biol. Cell 2003, 14, 3459-3469.

47. Kirchhausen, T. Three ways to make a vesicle. Nat. Rev. Mol. Cell Biol. 2000, 1, 187-198.

48. Kahn, R.A.; Cherfils, J.; Elias, M.; Lovering, R.C.; Munro, S.; Schurmann, A. Nomenclature for the human Arf family of GTP-binding proteins: ARF, ARL, and SAR proteins. J. Cell Biol. 2006, 172, 645-650.

49. Nakayama, K.; Wakatsuki, S. The structure and function of GGAs, the traffic controllers at the TGN sorting crossroads. Cell Struct. Func. 2003, 28, 431-442.

50. Robinson, M.S. Adaptable adaptors for coated vesicles. Trends Cell Biol 2004, 14, 167-174.

51. Bonifacino, J.S. The GGA proteins: Adaptors on the move. Nat. Rev. Mol. Cell Biol. 2004, 5, 23-32.

52. Whatmore, J.; Morgan, C.P.; Cunningham, E.; Collison, K.S.; Willison, K.R.; Cockcroft, S. ADP-ribosylation factor 1-regulated phospholipase D activity is localized at the plasma membrane and intracellular organelles in HL60 cells. Biochem. J. 1996, 320, 785-794. 
53. Honda, A.; Nogami, M.; Yokozeki, T.; Yamazaki, M.; Nakamura, H.; Watanabe, H.; Kawamoto, K.; Nakayama, K.; Morris, A.J.; Frohman, M.A.; Kanaho, Y. Phosphatidylinositol 4-phosphate 5-kinase alpha is a downstream effector of the small G protein ARF6 in membrane ruffle formation. Cell 1999, 99, 521-532.

54. Godi, A.; Pertile, P.; Meyers, R.; Marra, P.; di Tullio, G.; Iurisci, C.; Luini, A.; Corda, D.; de Matteis, M.A. ARF mediates recruitment of PtdIns-4-OH kinase-beta and stimulates synthesis of PtdIns(4,5)P2 on the Golgi complex. Nat. Cell Biol. 1999, 1, 280-287.

55. Shin, H.-W.; Nakayama, K. Dual control of membrane targeting by PtdIns(4)P and ARF. Trends Biochem. Sci. 2004, 29, 513-515.

56. Donaldson, J.G. Phospholipase D in endocytosis and endosomal recycling pathways. BBA-Mol. Cell Biol. L. 2009, 1791, 845-849.

57. Goldberg, J. Structural and functional analysis of the ARF1-ARFGAP complex reveals a role for coatomer in GTP hydrolysis. Cell 1999, 96, 893-902.

58. Gillingham, A.K.; Munro, S. The small G proteins of the Arf family and their regulators. Ann. Rev. Cell Dev. Biol. 2007, 23, 579-611.

59. Bielli, A.; Haney, C.J.; Gabreski, G.; Watkins, S.C.; Bannykh, S.I.; Aridor, M. Regulation of Sar1 NH2 terminus by GTP binding and hydrolysis promotes membrane deformation to control COPII vesicle fission. J. Cell Biol. 2005, 171, 919-924.

60. Lee, M.C.S.; Orci, L.; Hamamoto, S.; Futai, E.; Ravazzola, M.; Schekman, R. Sar1p N-terminal helix initiates membrane curvature and completes the fission of a COPII vesicle. Cell 2005, 122, 605-617.

61. Krauss, M.; Jia, J.Y.; Roux, A.; Beck, R.; Wieland, F.T.; de Camilli, P.; Haucke, V. Arf1-GTP-induced tubule formation suggests a function of Arf family proteins in curvature acquisition at sites of vesicle budding. J. Biol. Chem. 2008, 283, 27717-27723.

62. Beck, R.; Sun, Z.; Adolf, F.; Rutz, C.; Bassler, J.; Wild, K.; Sinning, I.; Hurt, E.; Brugger, B.; Bethune, J.; Wieland, F. Membrane curvature induced by Arf1-GTP is essential for vesicle formation. Proc. Natl. Acad. Sci. USA 2008, 105, 11731-11736.

63. Lundmark, R.; Doherty, G.J.; Vallis, Y.; Peter, B.J.; McMahon, H.T. Arf family GTP loading is activated by, and generates, positive membrane curvature. Biochem. J. 2008, 414, 189-194.

64. Long, K.R.; Yamamoto, Y.; Baker, A.L.; Watkins, S.C.; Coyne, C.B.; Conway, J.F.; Aridor, M. Sar1 assembly regulates membrane constriction and ER export. J. Cell Biol. 2010, 190, 115-128.

65. Farsad, K.; Ringstad, N.; Takei, K.; Floyd, S.R.; Rose, K.; de Camilli, P. Generation of high curvature membranes mediated by direct endophilin bilayer interactions. J. Cell Biol. 2001, 155, 193-200.

66. Gallop, J.L.; Jao, C.C.; Kent, H.M.; Butler, P.J.; Evans, P.R.; Langen, R.; McMahon, H.T. Mechanism of endophilin N-BAR domain-mediated membrane curvature. EMBO J. 2006, 25, 2898-2910.

67. Ford, M.G.; Mills, I.G.; Peter, B.J.; Vallis, Y.; Praefcke, G.J.; Evans, P.R.; McMahon, H.T. Curvature of clathrin-coated pits driven by epsin. Nature 2002, 419, 361-366. 
68. Takei, K.; Slepnev, V.I.; Haucke, V.; de Camilli, P. Functional partnership between amphiphysin and dynamin in clathrin-mediated endocytosis. Nat. Cell Biol. 1999, 1, 33-39.

69. Peter, B.J.; Kent, H.M.; Mills, I.G.; Vallis, Y.; Butler, P.J.G.; Evans, P.R.; McMahon, H.T. BAR domains as sensors of membrane curvature: The amphiphysin BAR structure. Science 2004, 303, 495-499.

70. Habermann, B. The BAR-domain family of proteins: a case of bending and binding? EMBO Rep. 2004, 5, 250-255.

71. Itoh, T.; de Camilli, P. BAR, F-BAR (EFC) and ENTH/ANTH domains in the regulation of membrane-cytosol interfaces and membrane curvature. BBA-Mol. Cell Biol. L. 2006, 1761, 897-912.

72. Masuda, M.; Mochizuki, N. Structural characteristics of BAR domain superfamily to sculpt the membrane. Semin. Cell Dev. Biol. 2010, 21, 391-398.

73. Suetsugu, S.; Toyooka, K.; Senju, Y. Subcellular membrane curvature mediated by the BAR domain superfamily proteins. Semi. Cell Dev. Biol. 2010, 21, 340-349.

74. Qualmann, B.; Koch, D.; Kessels, M.M. Let’s go bananas: Revisiting the endocytic BAR code. EMBO J. 2011, 30, 3501-3515.

75. Frost, A.; Unger, V.M.; de Camilli, P. The BAR domain superfamily: Membrane-molding macromolecules. Cell 2009, 137, 191-196.

76. Farsad, K.; De Camilli, P. Mechanisms of membrane deformation. Curr. Opin. Cell Biol. 2003, 15, 372-381.

77. Shimada, A.; Niwa, H.; Tsujita, K.; Suetsugu, S.; Nitta, K.; Hanawa-Suetsugu, K.; Akasaka, R.; Nishino, Y.; Toyama, M.; Chen, L.; et al. Curved EFC/F-BAR-domain dimers are joined end to end into a filament for membrane invagination in endocytosis. Cell 2007, 129, 761-772.

78. Frost, A.; Perera, R.; Roux, A.; Spasov, K.; Destaing, O.; Egelman, E.H.; de Camilli, P.; Unger, V.M. Structural basis of membrane invagination by F-BAR domains. Cell 2008, 132, 807-817.

79. Wang, Q.; Navarro, M.V.A.S.; Peng, G.; Molinelli, E.; Goh, S.L.; Judson, B.L.; Rajashankar, K.R.; Sondermann, H. Molecular mechanism of membrane constriction and tubulation mediated by the F-BAR protein Pacsin/Syndapin. Proc. Natl. Acad. Sci. USA 2009, 106, 12700-12705.

80. Henne, W.M.; Boucrot, E.; Meinecke, M.; Evergren, E.; Vallis, Y.; Mittal, R.; McMahon, H.T. FCHo proteins are nucleators of clathrin-mediated endocytosis. Science 2010, 328, 1281-1284.

81. Wu, M.; Huang, B.; Graham, M.; Raimondi, A.; Heuser, J.E.; Zhuang, X.; de Camilli, P. Coupling between clathrin-dependent endocytic budding and F-BAR-dependent tubulation in a cell-free system. Nat. Cell Biol. 2010, 12, 902-908.

82. Dawson, J.C.; Legg, J.A.; Machesky, L.M. Bar domain proteins: A role in tubulation, scission and actin assembly in clathrin-mediated endocytosis. Trends Cell Biol. 2006, 16, 493-498. 
83. Ferguson, S.; Raimondi, A.; Paradise, S.; Shen, H.; Mesaki, K.; Ferguson, A.; Destaing, O.; Ko, G.; Takasaki, J.; Cremona, O.; O’Toole, E.; de Camilli, P. Coordinated actions of actin and BAR proteins upstream of dynamin at endocytic clathrin-coated pits. Dev. Cell 2009, 17, 811-822.

84. Nie, Z.; Hirsch, D.S.; Luo, R.; Jian, X.; Stauffer, S.; Cremesti, A.; Andrade, J.; Lebowitz, J.; Marino, M.; Ahvazi, B.; et al. A BAR domain in the N terminus of the Arf GAP ASAP1 affects membrane structure and trafficking of epidermal growth factor receptor. Curr. Biol. 2006, 16, 130-139.

85. Zhu, G.; Chen, J.; Liu, J.; Brunzelle, J.S.; Huang, B.; Wakeham, N.; Terzyan, S.; Li, X.; Rao, Z.; Li, G.; Zhang, X.C. Structure of the APPL1 BAR-pH domain and characterization of its interaction with Rab5. EMBO J. 2007, 26, 3484-3493.

86. Soulet, F.; Yarar, D.; Leonard, M.; Schmid, S.L. SNX9 regulates dynamin assembly and is required for efficient clathrin-mediated endocytosis. Mol. Biol. Cell 2005, 16, 2058-2067.

87. Yarar, D.; Waterman-Storer, C.M.; Schmid, S.L. SNX9 couples actin assembly to phosphoinositide signals and is required for membrane remodeling during endocytosis. Dev. Cell 2007, 13, 43-56.

88. Pylypenko, O.; Lundmark, R.; Rasmuson, E.; Carlsson, S.R.; Rak, A. The PX-BAR membrane-remodeling unit of sorting nexin 9. EMBO J. 2007, 26, 4788-4800.

89. Cozier, G.E.; Carlton, J.; McGregor, A.H.; Gleeson, P.A.; Teasdale, R.D.; Mellor, H.; Cullen, P.J. The phox homology (PX) domain-dependent, 3-phosphoinositide-mediated association of sorting nexin-1 with an early sorting endosomal compartment is required for its ability to regulate epidermal growth factor receptor degradation. J. Biol. Chem. 2002, 277, 48730-48736.

90. Carlton, J.; Bujny, M.; Peter, B.J.; Oorschot, V.M.J.; Rutherford, A.; Mellor, H.; Klumperman, J.; McMahon, H.T.; Cullen, P.J. Sorting nexin-1 mediates tubular endosome-to-TGN transport through coincidence sensing of high-curvature membranes and 3-phosphoinositides. Curr. Biol. 2004, 14, 1791-1800.

91. Carlton, J.G.; Bujny, M.V.; Peter, B.J.; Oorschot, V.M.; Rutherford, A.; Arkell, R.S.; Klumperman, J.; McMahon, H.T.; Cullen, P.J. Sorting nexin-2 is associated with tubular elements of the early endosome, but is not essential for retromer-mediated endosome-to-TGN transport. J. Cell Sci. 2005, 118, 4527-4539.

92. Cullen, P.J. Endosomal sorting and signalling: an emerging role for sorting nexins. Nat. Rev. Mol. Cell Biol. 2008, 9, 574-582.

93. Van Weering, J.R.; Verkade, P.; Cullen, P.J. SNX-BAR proteins in phosphoinositide-mediated, tubular-based endosomal sorting. Semin. Cell Dev. Biol. 2010, 21, 371-380.

94. Van Weering, J.R.; Verkade, P.; Cullen, P.J. SNX-BAR-mediated endosome tubulation is co-ordinated with endosome maturation. Traffic 2012, 13, 94-107.

95. Man, Z.; Kondo, Y.; Koga, H.; Umino, H.; Nakayama, K.; Shin, H.W. Arfaptins are localized to the trans-Golgi by interaction with Arl1, but Not Arfs. J. Biol. Chem. 2011, 286, 11569-11578.

96. Lu, L.; Horstmann, H.; Ng, C.; Hong, W. Regulation of Golgi structure and function by ARF-like protein 1 (Arl1). J. Cell Sci. 2001, 114, 4543-4555. 
97. Lu, L.; Tai, G.; Hong, W. Autoantigen Golgin-97, an effector of Arl1 GTPase, participates in traffic from the endosome to the trans-Golgi network. Mol. Biol. Cell 2004, 15, 4426-4443.

98. Shin, H.-W.; Kobayashi, H.; Kitamura, M.; Waguri, S.; Suganuma, T.; Uchiyama, Y.; Nakayama, K. Roles of ARFRP1 (ADP-ribosylation factor-related protein 1) in post-Golgi membrane trafficking. J. Cell Sci. 2005, 118, 4039-4048.

99. Nishimoto-Morita, K.; Shin, H.W.; Mitsuhashi, H.; Kitamura, M.; Zhang, Q.; Johannes, L.; Nakayama, K. Differential effects of depletion of ARL1 and ARFRP1 on membrane trafficking between the trans-Golgi network and endosomes. J. Biol. Chem. 2009, 284, 10583-10592.

100. Miaczynska, M.; Christoforidis, S.; Giner, A.; Shevchenko, A.; Uttenweiler-Joseph, S.; Habermann, B.; Wilm, M.; Parton, R.G.; Zerial, M. APPL proteins link Rab5 to nuclear signal transduction via an endosomal compartment. Cell 2004, 116, 445-456.

101. Hua, Z.; Fatheddin, P.; Graham, T.R. An essential subfamily of Drs2p-related P-type ATPases is required for protein trafficking between Golgi complex and endosomal/vacuolar system. Mol. Biol. Cell 2002, 13, 3162-3177.

102. Liu, K.; Surendhran, K.; Nothwehr, S.F.; Graham, T.R. P4-ATPase requirement for AP-1/Clathrin function in protein transport from the trans-golgi network and early endosomes. Mol. Biol. Cell 2008, 19, 3526-3535.

103. Chantalat, S.; Park, S.K.; Hua, Z.; Liu, K.; Gobin, R.; Peyroche, A.; Rambourg, A.; Graham, T.R.; Jackson, C.L. The Arf activator Gea2p and the P-type ATPase Drs2p interact at the Golgi in saccharomyces cerevisiae. J. Cell Sci. 2004, 117, 711-722.

104. Jochum, A.; Jackson, D.; Schwarz, H.; Pipkorn, R.; Singer-Kruger, B. Yeast Ysl2p, homologous to Sec7 domain guanine nucleotide exchange factors, functions in endocytosis and maintenance of vacuole integrity and interacts with the Arf-like small GTPase Arl1p. Mol. Cell. Biol. 2002, 22, 4914-4928.

105. Wicky, S.; Schwarz, H.; Singer-Kruger, B. Molecular interactions of yeast neo1p, an essential member of the drs2 family of aminophospholipid translocases, and its role in membrane trafficking within the endomembrane system. Mol. Cell. Biol. 2004, 24, 7402-7418.

106. Takatsu, H.; Baba, K.; Shima, T.; Umino, H.; Kato, U.; Umeda, M.; Nakayama, K.; Shin, H.W. ATP9B, a P4-ATPase (a putative aminophospholipid translocase), localizes to the trans-golgi network in a cdc50 protein-independent manner. J. Biol. Chem. 2011, 286, 38159-38167.

(C) 2012 by the authors; licensee MDPI, Basel, Switzerland. This article is an open access article distributed under the terms and conditions of the Creative Commons Attribution license (http://creativecommons.org/licenses/by/3.0/). 